\title{
HELMINTOS INTESTINAIS EM CÃES DOMICILIADOS E ASPECTOS SOCIOECONÔMICOS E CULTURAIS DAS FAMÍLIAS PROPRIETÁRIAS DOS ANIMAIS DE LAGES, SC, BRASIL
}

\author{
Fernanda Magalhães Stalliviere ${ }^{1}$, Luciana Dalla Rosa ${ }^{2}$, Valdomiro Bellato ${ }^{1}$, Antonio \\ Pereira de Souza ${ }^{1}$, Amélia Aparecida Sartor ${ }^{1}$, Anderson Barbosa de Moura ${ }^{1}$ \\ 1 UDESC \\ 2 UFSM \\ Correspondência: Luciana Dalla Rosa: Iudallarosa@hotmail.com
}

\begin{abstract}
RESUMO: Este trabalho teve como objetivos determinar a prevalência de helmintos intestinais em cães domiciliados nas regiões central e periférica de Lages, SC, de correlacionar aspectos socioeconômicos e culturais das famílias proprietárias dos animais com a prevalência observada e de verificar a proporção entre as populações humana e canina. No período de dezembro de 2005 a dezembro de 2006, foram aplicados 600 questionários a pessoas residentes em cinco bairros da região central e cinco da região periférica da cidade de Lages, SC. Foram coletadas amostras de fezes de 523 cães, que foram processadas por técnicas de flutuação e sedimentação. Dados de faixa salarial e escolaridade foram utilizados para avaliação dos aspectos socioeconômicos e culturais, respectivamente. Para a mensuração das populações humana e canina foram computados todos os cães existentes nos domicílios. A prevalência para helmintos intestinais foi de $38,2 \%$, sendo maior em cães domiciliados na região periférica. O nível socioeconômico e cultural não foi significante para a prevalência de helmintos intestinais em cães. A proporção ser humano/cão foi de 3,5:1 e, para a cidade de Lages, foi estimada uma população de 44.780 cães domiciliados.
\end{abstract}

Palavras-chave: Canis familiaris; diagnóstico; endoparasitos; zoonoses

\section{INTESTINAL HELMINTHS IN DOMICILIATED DOGS AND SOCIOECONOMIC AND CULTURAL ASPECTS OF HOUSEHOLDS OWNING ANIMALS OF LAGES, SC, BRAZIL}

\begin{abstract}
This article had as objectives to determine the prevalence of intestinal helminths in dogs living in the central and peripheral areas of Lages, SC, to correlate socioeconomic and cultural aspects of households owning animals with the observed prevalence and determine the proportion between the human population and canine. From December 2005 to December 2006, 600 questionnaires were applied to people living in five districts of the central region and five in the peripheral region of the city of Lages. Stool samples were collected from 523 dogs, and were processed by flotation and sedimentation techniques. Data salary and education were used to evaluate the socioeconomic and cultural aspects, respectively. To assess the human and canine populations all dogs of each residence were computed. The prevalence of intestinal helminths was $38.2 \%$, higher from domestic dogs in the peripheral region. The socioeconomic and cultural levels were not significant for the prevalence of intestinal helminths in dogs. The proportion man/dog was 3.5:1. The domiciliated population of dogs in the city were estimated at 44,780 .
\end{abstract}

Key Words: Canis familiaris; diagnosis; endoparasites; zoonoses 


\section{INTRODUÇÃO}

Os animais de estimação, particularmente cães e gatos, proporcionam uma série de benefícios ao ser humano como companhia, guarda, auxílio a deficientes e idosos, e no desenvolvimento emocional e intelectual. Apesar dos benefícios, existem riscos de transmissão de enfermidades. Segundo Katagiri e Oliveira-Sequeira (2007), os últimos 20 anos foram marcados por um intenso progresso tecnológico e por importantes alterações culturais e sociais com reflexos tanto na saúde humana como animal. Entretanto, as zoonoses, por exemplo, Larva Migrans Cutânea e Larva Migrans Visceral, continuam sendo um problema de saúde pública. $O$ conhecimento da fauna parasitária dos animais domésticos torna-se necessário para atuar na prevenção das doenças parasitárias.

O presente trabalho foi realizado com os objetivos de determinar e comparar a prevalência de helmintos intestinais em cães domiciliados, das regiões central e periférica, da cidade de Lages, SC, de correlacionar aspectos socioeconômicos e culturais das famílias proprietárias dos animais com a prevalência e de verificar a proporção entre as populações humana e canina.

\section{MATERIAL E MÉTODOS}

A cidade de Lages está situada no Planalto Serrano do estado de Santa Catarina, com altitude de $961 \mathrm{~m}$, latitude de $27^{\circ} 48^{\prime} S$ e longitude de $50^{\circ} 20^{\prime} \mathrm{O}$. O clima é subtropical com temperatura média de $14,3^{\circ} \mathrm{C}$, com a mínima de $-7,4^{\circ} \mathrm{C}$ e a máxima de $35^{\circ} \mathrm{C}$, umidade relativa média de 79,3\% (PML, 2007). A população aproximada da cidade é de 156.727 habitantes, distribuídas em 68 bairros (IBGE, 2010).

Para este estudo, a cidade foi dividida em duas regiões: central e periférica. Foram incluídos por sorteio, 10 bairros, sendo cinco localizados na região central e cinco na região periférica, perfazendo, aleatoriamente, um total de 600 domicílios.

Os dados foram coletados por meio de entrevista estruturada onde foram considerados critérios de inclusão na pesquisa a concordância em participar da investigação e responder ao questionário formulado.

Aos entrevistados que possuíam animais, foram esclarecidos os procedimentos para coleta de dados. Após preenchimento de uma ficha clínica para cada animal, foi realizado o exame clínico. As amostras de fezes, recentemente eliminadas, foram recolhidas, acondicionadas em sacos plásticos, identificadas, mantidas em caixas de isopor com gelo e transportadas ao Laboratório de Parasitologia e Doenças Parasitárias da Universidade do Estado de Santa Catarina, onde foram mantidas sob refrigeração entre 2 e $8^{\circ} \mathrm{C}$. Nas residências onde não houve coleta de fezes dos animais, estes foram contabilizados na amostragem para estabelecer a proporção ser humano/cão. As amostras de fezes foram analisadas pelas técnicas parasitológicas de Willis (1921), Gordon e Whitlock (1939) modificada e de Dennis et al. (1954) modificada.

Os dados foram analisados pelo Teste de $x^{2}$ a fim de verificar a significância entre as variáveis qualitativas e regressão logística, utilizando-se os programas SPHINX ${ }^{\circledR}$ e SAS (SAS Institute, 1999), respectivamente. Para as análises qualitativas, foi considerado $P<0,01$, e para testes de regressão logística $P<0,01$ e $P<0,05$. Nas faixas etárias onde não houve animal positivo para o gênero Toxocara, atribuiu-se valor 0,5 de positividade, conforme proposto por Shafii e Price (2007). 

familias proprietárias dos animais de Lages, SC, Brasil

\section{RESULTADOS E DISCUSSÃO}

Das 600 residências visitadas, em 369 foi verificada a presença de cães domiciliados, totalizando 622 animais. Foram coletadas amostras de fezes de 523 cães $(84,1 \%)$, com prevalência de helmintos intestinais de $38,2 \%$. Nos bairros centrais, foram coletadas amostras de 269 cães, sendo 51 (19\%) positivas para helmintos intestinais e nos bairros periféricos, de 254 amostras, 149 (58,7\%) estavam positivas $(\mathrm{P}<0,01)$. Diferentes resultados foram observadas por Leite et al. (2004) e Vasconcellos et al. (2006), com $45,08 \%$ e $45,6 \%$ de positividade em cães domiciliados e mantidos em canil municipal, respectivamente. O parasitismo em cães domiciliados geralmente é menor que em cães com acesso à rua, todavia, os resultados observados em cães domiciliados da região periférica da cidade de Lages, se aproximam aos observados por Blazius et al. (2005) em Itapema, SC, em cães errantes. Além da procedência, deve-se considerar que as prevalências variam, normalmente, devido a fatores climáticos, idade dos animais, hábitos culturais dos proprietários e dos recursos diagnósticos.

Os dados de prevalência de infecções por helmintos constam na tabela 1. A prevalência observada para o gênero Ancylostoma spp. foi superior a encontrada por Mundin et al. (2001), na cidade de Uberlândia, MG, que observaram, em cães domiciliados, positividade de $5,71 \%$ para ancilostomatídeos e inferior aos resultados de Oliveira-Sequeira et al. (2002) em Botucatu, SP, que verificaram $23,6 \%$ das amostras fecais parasitados por Ancylostoma spp.. Para o gênero Toxocara sp. a prevalência observada foi semelhante às verificadas por Oliveira-Sequeira et al. (2002), de 5,5\%, e aos resultados observados por Brener et al. (2005), onde a segunda espécie mais encontrada no estudo foi $0 T$. canis, observado em $7,1 \%$ das amostras. A prevalência para Trichuris $\mathrm{sp}$. foi superior à verificada por Leite et al. (2004), em Curitiba, PR, que constataram prevalência de 3,3\%. Para Dipylidium caninum, os resultados verificados corroboram os de Gennari et al. (1999) que também encontraram baixas porcentagens. Esta prevalência pode estar associada à frequência do hospedeiro intermediário (pulgas), que na avaliação clínica dos animais esteve presente em somente $22,9 \%$ dos animais (Stalliviere et al., 2009). Foi constada pela primeira vez na cidade de Lages, SC, a presença de Oncicola sp. Este gênero de helminto foi relatado por Soulsby (1987) parasitando cães e por Henke et al. (2002) parasitando coiotes (Canis latrans).

\begin{tabular}{|c|c|c|}
\hline \multirow{2}{*}{ Gêneros } & \multicolumn{2}{|c|}{ Cães } \\
\hline & Positivos (n) & Prevalência (\%) \\
\hline Ancylostoma spp. & 64 & 12,2 \\
\hline Toxocara sp. & 27 & 5,1 \\
\hline Trichuris sp. & 42 & 8,0 \\
\hline Dipylidium caninum & 05 & 1,0 \\
\hline Família Taeniidae & 01 & 0,2 \\
\hline Ancylostoma spp. + Toxocara sp.+ Trichuris sp. & 08 & 1,5 \\
\hline Ancylostoma spp. + Toxocara sp. & 15 & 2,9 \\
\hline Ancylostoma spp. + Trichuris sp. & 32 & 6,1 \\
\hline Toxocara sp. + Trichuris sp. & 04 & 0,7 \\
\hline Ancylostoma spp. + Toxocara sp.+ Taeniidae & 01 & 0,2 \\
\hline $\begin{array}{l}\text { Dipylidium caninum + Oncicola sp. } \\
\text { Total }\end{array}$ & $\begin{array}{c}01 \\
200\end{array}$ & $\begin{array}{r}0,2 \\
38,2\end{array}$ \\
\hline
\end{tabular}

A idade média dos animais parasitados foi de 3,6 anos. Para Ancylostoma spp. foi de 3,6, para Toxocara sp. 2,5 e para Trichuris sp. 4,2 anos. Segundo Vasconcellos et al. (2006) os animais com menos de um ano de idade apresentam maiores taxas de infecções. Para Ramírez-Barrios et al. (2004), as infecções parasitárias acometem cães de todas as idades, mas são mais prevalentes em filhotes, principalmente porque muitos parasitos utilizam vias de transmissão que expõem especificamente recémnascidos ou neonatos e também porque os cães jovens não respondem imunologicamente de forma eficaz. No presente trabalho, foi observada 
correlação $(P<0,01)$ entre a idade dos animais e a positividade para Toxocara sp., com maiores percentuais entre os cães de até seis meses (Tabela 2).

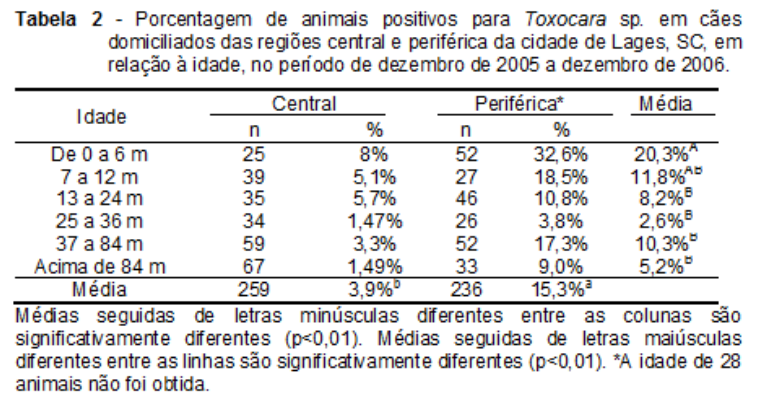

O número médio de ovos por grama de fezes (OPG), observado nos cães da região central foi 36,43 para Ancylostoma spp., 38,66 para Trichuris sp. e 56,50 para Toxocara sp., e na região periférica foram 415,35 para Ancylostoma spp., 265,35 para Trichuris sp. e 138,97 para Toxocara sp. O OPG fornece informações importantes acerca do grau de contaminação ambiental permitindo prever que em condições ambientais favoráveis haverá o desenvolvimento de formas infectantes e a possibilidade de transmissão dos agentes.

O conhecimento de que enfermidades podem ser transmitidas pelos animais foi relatado por $93,3 \%$ dos entrevistados e destes, $39,6 \%$ afirmaram ter recebido orientação do médico veterinário quanto à transmissão de doenças ao ser humano. As enfermidades mais citadas foram sarna $(34,7 \%)$ bicho-do-pé (34,1\%), toxoplasmose (15\%) e bicho geográfico $(11,2 \%)$. Dos proprietários de cães, $46,7 \%$ não levaram 0 animal ao veterinário, 32,1\% levaram uma vez no ano, $14,9 \%$ mensalmente e $6,3 \%$ semanalmente. Dos moradores que tiveram acesso aos serviços veterinários, 48\% afirmaram não ter recebido nenhuma orientação sobre zoonoses. Na Austrália, Bugg et al. (1999) constataram que $62,5 \%$ dos proprietários tinham conhecimento sobre enfermidades que podem ser transmitidas pelos animais. Em estudos realizados na Índia, Traub et al. (2005), verificaram que dos entrevistados, aproximadamente $50 \%$ estavam em contato frequente com cães e destes, $6 \%$ possuíam conhecimento de zoonoses parasitárias.

O uso de anti-helmínticos em humanos foi citado por $63,8 \%$ dos entrevistados. Grande parte dos moradores relatou visitar o posto de saúde e utilizar a medicação frequentemente em crianças. Já em cães, o uso de anti-helmínticos, nos últimos 12 meses, foi relatado por $78,3 \%$ dos proprietários. O percentual de uso, informado pelos proprietários dos bairros da região central, foi de $85,8 \%$ e dos bairros da região periférica, $73,0 \%$. Estes percentuais justificam, em parte, a diferença $(\mathrm{P}<0,01)$ entre as prevalências observadas nas duas regiões da cidade. Muradian et al. (2005) em São Paulo, SP, verificaram que $58,5 \%$ dos animais examinados haviam recebido anti-helmínticos pelo menos uma vez na vida, e destes $41,7 \%$ estavam positivos para $T$. canis.

Os locais de defecação dos animais, informado pelos proprietários, foram: $62,9 \%$ no gramado; $19,5 \%$ no jardim com piso ou canil; 9,2\% não possuem local próprio e 8,4\% afirmaram que seus animais defecam em outros locais. Dos cães domiciliados 68,5\% permanecem no pátio, canil ou dentro da casa. Habluetzel et al. (2003), avaliando variáveis semelhantes, constataram que $29,6 \%$ dos cães defecam no campo, $25,3 \%$ em canis, $19,6 \%$ em jardins, $6,3 \%$ em outros locais, $10,9 \%$ nas ruas e $8,4 \%$ em praças. O hábito de recolher as fezes de seus animais diariamente foi relatado em $64 \%$ dos domicílios, diferindo estatisticamente daqueles que recolhiam semanalmente, mensalmente e não recolhiam ou o faziam de outras formas $(P<0,01)$. Bugg et al. (1999) afirmaram que $56 \%$ dos proprietários 

familias proprietárias dos animais de Lages, SC, Brasil

recolhem as fezes do quintal de quatro a cinco vezes por semana. Ao recolher a defecação, o proprietário evita que as fezes contaminadas permaneçam no ambiente, criando condição favorável ao desenvolvimento de formas infectantes de helmintos e infecção de pessoas.

Os níveis de infecções por helmintos dos animais da região periférica foram maiores $(P<0,01)$, quando comparados com os da região central. Dos proprietários de cães, $2,73 \%$ não eram alfabetizados; $47,27 \%$ possuíam ensino fundamental; 27,01\% ensino médio e $22,99 \%$ possuíam ensino superior. Dos cães positivos, $58,6 \%$ pertenciam a proprietários com ensino fundamental e destes 84,2\% residiam na região periférica da cidade. Comparando as diferentes faixas salariais das famílias e o nível de escolaridade, entre as duas regiões, não houve diferença estatística. Diante disso, é possível que fatores como educação em saúde, o hábito de recorrer aos serviços veterinários, manter um controle sanitário de seus animais e a manutenção dos mesmos em locais adequados, possam explicar as diferenças constatadas.

Nas 600 residências, foi constatada a presença de 2187 pessoas (3,6 pessoas/domicílio) e foram cadastrados 622 cães, relação de 1,04 cão/domicílio. Considerando a população urbana da cidade de Lages, SC, de aproximadamente 156.727 habitantes (IBGE, 2010) e a relação pessoa/cão (3,5:1), a população canina da cidade de Lages, SC, foi de aproximadamente $\quad 44.780 \quad$ cães domiciliados. Diferente disto, Dias et al. (2004), em Taboão da Serra, SP, observaram 0,84 cães por domicílio e a razão entre a população humana $e$ canina foi de 5,14:1. Já Muradian et al. (2005), em São Paulo, SP, relataram a relação ser humano/cão de 12,5:1 e 0,35 cão/residência. A correta avaliação dos parâmetros populacionais de cães, nas diversas regiões e municípios do País, permite melhorar as tomadas de decisões tanto econômicas como de saúde por parte de empresários e do governo.

O número médio de crianças por domicílio foi de 0,71 , com a presença de pelo menos uma criança em $32 \%$ dos domicílios. Este percentual evidencia a importância de ações preventivas a fim de evitar a transmissão de agentes parasitários responsáveis por zoonoses, principalmente devido aos hábitos incipientes de higiene das crianças, no contato com animais e/ou suas excretas.

\section{CONCLUSÃO}

A prevalência para helmintos intestinais em cães domiciliados na região periférica da cidade de Lages, neste experimento, é maior que na região central, e os principais agentes etiológicos são Ancylostoma spp. Toxocara sp. e Trichuris sp. Embora as populações residentes nos bairros da região periférica encontrem-se mais expostas aos agentes com potencial zoonótico, os níveis socioeconômicos e culturais não apresentaram correlação com a prevalência de endoparasitos em cães.

\section{NOTAS INFORMATIVAS}

O presente trabalho foi aprovado pelos Comitês de Ética em Pesquisa com Seres Humanos (CEPSH) da Universidade do Estado de Santa Catarina - UDESC (no. de Referência 170/05) e de Ética em Experimentação Animal da UDESC - CETEA (Protocolo no. 1.10/05 e 1.20/06).

\section{REFERÊNCIAS}

BLAZIUS, R.D.; EMERICK, S.; PROPHIRO, J.S. et al. Ocorrência de protozoários e helmintos em amostras de fezes de cães 
errantes da cidade de Itapema, SC. Revista da Sociedade Brasileira de Medicina Tropical, v.38, n.1, p.73-74, 2005.

BRENER, B.; LISBOA, L.; MATTOS, D.P.B.G. et al. Frequência de endoparasitas em amostras fecais de cães e gatos dos municípios do Rio de Janeiro e Niterói. Revista Brasileira de Ciências Veterinárias, v.12, n.1-3, p.102-105, 2005.

BUGG, R.J.; ROBERTSON, I.D.; ELLIOT, A.D. et al. A gastrointestinal parasites of urban dogs in Perth, Western Australia. Veterinary Journal, v.157, n.3, p.295-301, 1999.

DENNIS, W.R.; STONE, W.M.; SWANSON, L.E. A new laboratory and field diagnostic test for fluke ova in feces. Journal of the American Veterinary Medical Association, v.124, n.922, p.47-50, 1954.

DIAS, R.A.; GARCIA, R.C.; SILVA, D.F. et al. Estimativa de populações canina e felina domiciliadas em zona urbana do Estado de São Paulo. Revista de Saúde Pública, v.38, n.4, p.565-570, 2004.

GENNARI, S.M.; KASAI, N.; PENA, H.F.J. et al. Ocorrência de protozoários e helmintos em amostras de fezes de cães e gatos da cidade de São Paulo. Brazilian Journal of Veterinary Research and Animal Science, v.36, n.2, p. 791, 1999.

GORDON, H.McL.; WHITLOCK, H.V. A new technique for counting nematode eggs faeces. Journal of the Commonwealth Science and Industry Organization, v.12, n.1, p 50-52,1939.

HABLUETZEL, A.; TRALDIA, G.; RUGGIERI, S. et al. An estimation of Toxocara canis prevalence in dogs, environmental egg contamination and risk of human infection in the Marche region of Italy. Veterinary Parasitology, v.113, n.3-4, p.243-252, 2003.

HENKE, S.E.; PENCE, D.B.; BRYANT, F.C. et al. Effect of short-term coyote removal on populations of coyote helminthes. Journal of Wildlife Diseases, v.38, n.1, p.54-67, 2002.

IBGE - Instituto Brasileiro de Geografia e Estatística - Sinopse do Censo Demográfico 2010. Disponível em:

<http://www.ibge.gov.br/home/estatistica/popula cao/censo2010/tabelas_pdf/Santa_catarina.pdf> Acesso em: 30/08/2012.

KATAGIRI, S.; OLIVEIRA-SEQUEIRA, T.C.G. Zoonoses causadas por parasitas intestinais de cães e o problema do diagnóstico. Arquivo Instituto Biológico, v.74, n.2, p.175-184, 2007.
LEITE, L.C.; MARINONI, L.P.; CÍRIO, S.M. et al. Endoparasitas em cães (Canis familiares) na cidade de Curitiba - Paraná - Brasil. Archives of Veterinary Science, v 9, n.2, p.95-99, 2004.

MUNDIN, M.J.S.; CABRA, D.D.; FARIA, E.S.M et al. Endoparasitas de importância como zoonoses em fezes de cães domiciliados de Uberlândia, Minas Gerais. Veterinária Notícias, v.7, n.2, p.73-77, 2001.

MURADIAN, V.; GENNARI, S.M.; GLICKMAN, L.T. et al. Epidemiological aspects of Visceral Larva Migrans in children living at São Remo Community, São Paulo (SP), Brazil. Veterinary Parasitology, v.134, n.1-2, p.93-97, 2005.

OLIVEIRA-SEQUEIRA, T.C.G.; AMARANTE, A.F.T.; FERRARI, T.B. et al. Prevalence of intestinal parasites in dogs from São Paulo, Brazil. Veterinary Parasitology, v.103, n.1-2, p.19-27, 2002.

PML. Prefeitura Municipal de Lages. Disponível em: < http://www.lages.sc.gov.br/>. Acesso em 22/01/2007.

RAMÍREZ-BARRIOS, R. A.; BARBOZA-MENAB, G.; MUÑOZ, J. et al. Prevalence of intestinal parsites in dogs under veterinary care in Maracaibo, Venezuela. Veterinary

Parasitology, v.121, n.1-2, p.11-20, 2004.

SAS INSTITUTE. SAS/STAT User's Guide 8.0. Cary-NC: SAS Institute, 1999.

SHAFII, B.; PRICE, W. J. SAS Workshops. Proc Genmod: College of Agriculture, 2007.

SOULSBY, E. J. L. Parasitologia y Enfermedades parasitarias en los animales domésticos. 7 ed. México: Interamericana, 1987. 823p.

STALLIVIERE, F.M.; BELLATO, V.; SOUZA, A.P. et al. Ectoparasitos em Canis familiaris da cidade de Lages, SC, Brasil e aspectos sócioeconômicos e culturais das famílias dos proprietários dos animais. Revista de Ciências Agroveterinárias, v.8, n.2, p.179-183, 2009.

TRAUB, R.J.; ROBERTSON, I.D.; IRWIN, P.J. et al. A Canine gastrointestinal parasitic zoonoses in India. Trends in Parasitology, v.21, n.1, p.42-48, 2005.

VASCONCELLOS, M.C.; BARROS, J.S.L.; OLIVEIRA, C.S. Parasitas gastrointestinais em cães institucionalizados no Rio de Janeiro, RJ. Revista de Saúde Pública, v.40, n 2, p.321323, 2006.

WILLIS, H.H. A simple levitation method for detection of hookworm ova. Medical Journal of Australia, v.8, p.375-376, 1921. 